\title{
Ligand Based Pharmacophore Modeling, Virtual Screening, and Molecular Docking Studies of Asymmetrical Hexahydro-2H-Indazole Analogs of Curcumin (AIACs) to Discover Novel Estrogen Receptors Alpha (ERa) Inhibitor
}

\author{
Hariyanti $^{1}$, Kusmardi ${ }^{2}$, Arry Yanuar $^{3}$, and Hayun ${ }^{3, *}$ \\ ${ }^{1}$ Faculty of Pharmacy and Science, Universitas Muhammadiyah Prof. DR. HAMKA, \\ Jl. Delima II/IV, Duren Sawit, East Jakarta 13460, Indonesia \\ ${ }^{2}$ Faculty of Medicine, Universitas Indonesia, UI Salemba Campus, \\ Jl. Salemba Raya No. 6, Central Jakarta 10430, Indonesia \\ ${ }^{3}$ Faculty of Pharmacy, Universitas Indonesia, Depok 16424, West Java, Indonesia
}

\section{* Corresponding author:}

email:hayun@farmasi.ui.ac.id

Received: March 8, 2020

Accepted: September 17, 2020

DOI: $10.22146 / \mathrm{ijc} .54745$

\begin{abstract}
The estrogen receptor alpha (ER $\alpha)$ plays an important role in breast development and pro-proliferation signal activation in the normal and cancerous breast. The ER $\alpha$ inhibitors were potentially active as cytotoxic agents against breast cancer. This study was conducted in order to find Asymmetrical Hexahydro-2H-Indazole Analogs of Curcumin (AIACs) as hits of ER $\alpha$ inhibitor. A training set of 17 selected ER $\alpha$ inhibitors was used to create 10 pharmacophore models using LigandScout 4.2. The pharmacophore models were validated using 383 active compounds as positive data and 20674 decoys as negative data obtained from DUD.E. Model 2 was found as the best pharmacophore model and consisted of three types of pharmacophore features, viz. one hydrophobic, one hydrogen bond acceptor, and aromatic interactions. Model 2 was utilized for ligandbased virtual screening 186 of AIACs, AMACs, intermediates, and Mannich base derivative compounds. The hits obtained were further screened using molecular docking, analyzed using drug scan, and tested for its synthesis accessibility. Fourteen compounds were fulfilled as hits in pharmacophore modeling, in which 10 hits were selected by molecular docking, but only seven hits met Lipinski's rule of five and had medium synthesis accessibility. In conclusion, seven compounds were suggested to be potentially active as ER inhibitors and deserve to be synthesized and further investigated.
\end{abstract}

Keywords: asymmetric hexahydro-2H-indazole analogs of curcumin; AIACs; estrogen receptor alpha inhibitor; ER $\alpha$ inhibitor; pharmacophore modeling; molecular docking; breast cancer

\section{- INTRODUCTION}

Breast cancer is a disease that occurs almost entirely in women. It is the second leading cause of death by a disease [1]. In 2018, 2.1 million new cases of breast cancer were found. In most countries, this disease was the most commonly diagnosed cancer (154 out of 185) [2]. The estrogen receptor alpha (ER $\alpha)$ plays a role in breast development and the activation of the pro-proliferation signal in normal and cancerous breasts [3]. The growth of breast cancer cells is characterized by the high expression of the receptors [4]. Nowadays, ER $\alpha$ has been developed and tested as molecular targets for the treatment and prevention of breast cancer [5].

Monocarbonyl Analogs of Curcumin (MACs) and Asymmetrical Monocarbonyl Analogs of Curcumin (AMACs) were reported to show better inhibition against cancer cell proliferation of SMMC-7221, MCF7, and PC-3 compared to curcumin [6]. Diethylamine Mannich base substitution of the phenyl ring of MACs showed increased activity and selectivity of its anticancer properties [7]. Mannich base substitution of AMACs

Hariyanti et al. 
also showed cytotoxicity potential against HeLa, MCF-7, and WiDr cells [8-9]. The Mannich base acted as an important pharmacophore group in high-potential drugs [10].

Several studies reported that structural modification of symmetrical MACs into symmetrical Hexahydro-2HIndazole Analog (IAC) exhibited good antioxidant and antitumor activity against Hep G2, WI38, VERO, and MCF-7 cells [11-12]. Some studies also reported that modified compounds with indazole group formation show better anticancer activity [13-14]. However, to the best of our knowledge, there were no reports about the development of AMACs into AIACs and its derivatives. In the present study, we designed 186 structures of AIACs, their derivatives, and intermediate compounds that have different substituents in one of the benzene rings.

In this study, the initial virtual screening of 186 ligand designs was carried out using the Ligand-based virtual screening (LBVS) method. The LBVS methods compare a library of compounds with a known active ligand. Two notable advantages of LBVS methods are that they do not require structural information of a target receptor and that they are faster than structure-based methods [15]. The objective of the study was to discover a new molecular entity of AIACs compounds as hits for ER $\alpha$ inhibitor. The 186 AIACs, AMACs, intermediates, and the Mannich base derivative compounds were screened virtually through ligand-based pharmacophore modeling, structure-based molecular docking, analysis by drug scan, and tested for its synthesis accessibility.

\section{- COMPUTATIONAL METHODS}

\section{Equipment}

The hardware used for the calculations, pharmacophore modeling, and molecular docking was a laptop with the following specification: DesktopAF57S8U, Processor Intel(R) Core(TM) i5-5200 CPU@ $2.20 \mathrm{GHz} 2.20 \mathrm{GHz}$, RAM $16 \mathrm{~GB}$, Operating System Windows 10, 64 bit, Graphic Card AMD Radeon R9M275 4GB. The software used includes Marvinsketch, LigandScout 4.2, and AutoDockTools (v 4.2) integrated LigandScout software 4.2.

\section{Procedure}

\section{Data preparation}

The 186 compounds of Asymmetrical Hexahydro2H-Indazole Analogs of Curcumin (AIACs), AMACs, its intermediate, and Mannich base derivatives were drawn using Marvinsketch (www.chemaxon.com). The structures are shown in Table S1.a-g. A set of data of 34 ERa inhibitor compounds (Table S2.a-b) that consists of four native ligands of ER PDB and 30 other compounds with $\mathrm{pIC}_{50}$ values in the range of 4.40 to 9.86 , were obtained from www.pubchem.com. The threedimensional (3D) Estrogen Homo sapiens receptor alpha $(\mathrm{ER} \alpha)$ in the complex with E4D600 ligands (PDB code: 1SJ0) was obtained online from a database: NCBI, Research Collaboratory for Structural Bioinformatics Protein Data Bank http://www.pdb.org/pdb/home/ $[9,16]$.

\section{Pharmacophore models preparation and validation}

The pharmacophore models were created using LigandScout4.2 [17]. A set of data of $34 \mathrm{ERa}$ inhibitor compounds were grouped according to their cluster of chemical structure similarity. Every cluster of the compounds found was divided in the same proportion randomly to obtain two groups that consist of 17 molecules of ERa inhibitors. Seventeen selected molecules were used as a training set to create ten pharmacophore models. The 383 active compounds and 20674 decoys were used as positive and negative data to validate the pharmacophore models and determine the best pharmacophore model. The validation parameter of the receiver operating characteristic (ROC) that consisted of areas under the curves (AUC 100\%) and enrichment factors (EF 1\%) was calculated to determine the sensitivity, specificity, and accuracy values. The pharmacophore model with sensitivity $>0.5$, specificity $>0.5$, AUC value $>0.7$, and a hit score $>0.7$ was used as a virtual screening model [18].

\section{Ligand-based virtual screening}

The virtual screening was used to find AIACs compounds as hits of ERa inhibitors. A database of 186 AIACs and AMACs compounds in .mol file format was put in a screening database of a selected and validated pharmacophore model, then the screening process was 
performed until completed. Tamoxifen was used as a positive control. The hit compounds obtained were further sorted based on the best pharmacophore fit values.

\section{Molecular docking study}

Docking simulations were carried out to visualize molecular-level interactions between the hits obtained from ligand-based pharmacophore modeling with the active site of ERa (PDB code: 1SJ0) using tamoxifen as a positive control. The docking was done using AutoDock (v4.2) (autodock.scripps.edu/resources/autodock-force field) integrated with LigandScout. The method was validated by extracting the co-crystalline ligand (E4D600) from the ERa crystallographic structure and re-docking the copy of the ligand into its active site. The root-meansquare deviation (RMSD) value of the copy ligand conformation docked at the receptor as compared to the co-crystalline ligand conformation at the same receptor was calculated. The molecular docking was performed by running the Genetic Algorithm parameters 100 times, with algorithm generation number of 27,000, 2,500,000 energy evaluation numbers, and 150 population.

\section{Drug scan and synthesis accessibility analysis}

The drug scan and synthesis accessibility were analyzed online on a website (http://swissadme.ch). The analysis involved uploading the ligand file in smile format. Then, the results were downloaded in excel format.

\section{- RESULTS AND DISCUSSION}

The asymmetrical hexahydro- $2 \mathrm{H}$-indazole curcumins (AIACs) were designed as the development of AMACs that refers to the modification of MACs into a symmetrical hexahydro- $2 \mathrm{H}$-indazole analog of curcumins (IACs) that provided good results for activity in several cancer cells including breast cancer cells [12]. The AMACs and derivatives exhibited cytotoxicity potential against HeLa, MCF-7, and WiDr cell lines [8-9], thus AIACs were also predicted to have cytotoxic activities as well. The structure of the designed AIACs (Table S1.c-f) had different substituents in one of the benzene rings $\left(-\mathrm{H},-\mathrm{CH}_{3},-\mathrm{F},-\right.$ $\mathrm{Cl},-\mathrm{OCH}_{3}$, -dimethoxy) by considering their different characteristics of electronegativity, electronic charge, and induction effect of the substituents resulting in different geometric shapes for each analog compound. Therefore, the structures had variations in the bonding interactions with the receptors. In the present study, the AIACs were first screened virtually by ligand-based pharmacophore modeling. The hits obtained were then screened by structure-based modeling using molecular docking and further screened again by drug scan and synthesis accessibility analysis to obtain the new bioactive compounds as hits of ERa inhibitor.

Virtual screening (VS) has emerged as a crucial device in identifying bioactive compounds via computational means by employing knowledge on the protein target or known bioactive ligands [19]. Several VS with $\mathrm{ER} \alpha$ as a protein target using the structure-based virtual screening (SBVS) protocol had been reported. The protocol screened compounds based on the interactions of the 3D structure of the compounds with the target protein [20-21]. In this study, before the compounds were screened with the SBVS protocol, the compounds were first screened using the LBVS protocol. The compounds were selected based on the similarity of the molecular structure (in terms of shape, pharmacophoric features, molecular fields, etc.), which was believed to show similar behavior. LBVS techniques that consist of substructure mining and fingerprint searches are faster than SBVS techniques (e.g., molecular docking) [22-24]. The benefit of combining docking primarily based digital screening with pharmacophore-primarily based digital screening is that the database of ligands can be pre-filtered by using a pharmacophore query, before assessment using docking simulations. The docking simulations can be published and filtered with the use of a pharmacophore question to dispose of any compounds that fail to bind consistently with the pharmacophore query. The pharmacophore version can in this case be used for the position of the ligand, in addition to the precision of a molecule towards the pharmacophore question; or to guide the placement via a constraint while scoring the extraordinary docking poses [25].

\section{Pharmacophore Model Preparation and Validation}

The 3D pharmacophore of the various training sets produced 10 pharmacophore models. The validation of the models by a data set of 383 active compounds as

Hariyanti et al. 
positive data and 20674 decoys as negative data produced hit scores in the range of $0.7679-0.7718$ and three pharmacophore features marked by red, yellow, and purple for HBA, hydrophobic, and AR interaction, respectively. The HBA interaction was formed by the hydroxyl groups in the AR and the ether group; the hydrophobic interaction was formed by the AR, which also showed AR interactions. Fig. 1 presents the $3 \mathrm{D}$ pharmacophore model 2 with the type of features and distance between features. The $3 \mathrm{D}$ and $2 \mathrm{D}$ pharmacophores model of training set E4D600, is shown in Fig. 2, and the types of pharmacophore features marked by color differences in the training set compound are shown in Table 1. The ROC curve of model 2 is shown in Fig. 3. The screening of the ERa inhibitors using model 2 pharmacophore produced the best result in sensitivity = 0.687 ; specificity $=0.845$; AUC $100 \%=0.80$; accuracy $=$ $0.843 ; \mathrm{EF}_{1 \%}=26.7$ and hit score $=0.7712$. The set of five hypotheses with sensitivity $>0.5$, specificity $>0.5$, AUC value $>0.7$, and hit score $>0.7$ can be used as a virtual screening model [26]. The EF and AUC values showed that the virtual screening method using pharmacophore model 2 was an excellent screening model. The EF and AUC values were worse than the SBVS protocol reported by Yuniarti et al. [27], but it was still better than the results reported by Setiawati et al. [28], and also the EF and AUC values of the SBVS protocol used to identify ligands for ERa in DUD-E (EF = 15.4, AUC = 0.675) [29]. Therefore, the virtual screening of 186 AIACs and AMACs compounds was performed using model 2 .

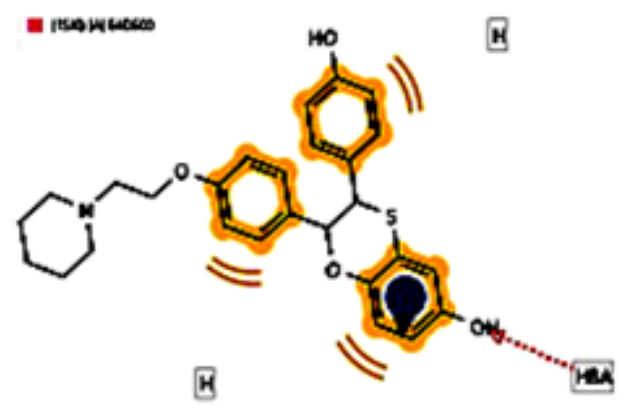

ㅂ.

(a)

\section{Ligand-Based Virtual Screening}

The key features of the pharmacophore interactions of tamoxifen on ERa were hydrophobic interactions, HBAs, and AR interactions (Fig. 1). The virtual screening of 186 AIACs and AMACs compounds resulted in 14 hit compounds that are shown in Table 2. The pharmacophore fit values measured geometric features of molecules for 3D structure-based pharmacophore models. The higher the pharmacophore fit values indicated the higher possibility of the hit to match with the pharmacophore model and show higher activity as ERa inhibitors. The pharmacophore fit values of 14 hits ranged from 45.32 to 53.43 . Compounds 3B8,

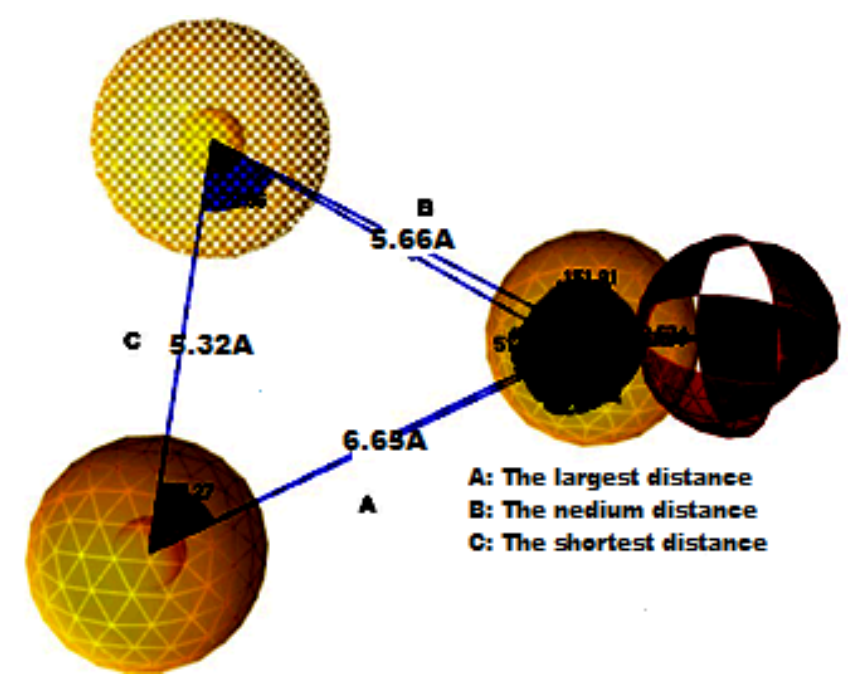

Fig 1. The pharmacophore model 2 features and the distance between features obtained by the LigandScout 4.2 software

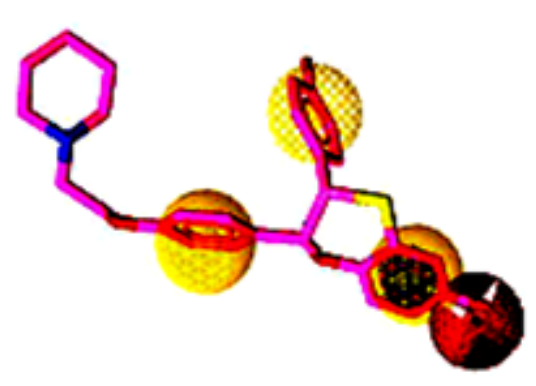

(b)

Fig 2. Pharmacophore (a) 2D and (b) 3D models of E4D600 obtained by the LigandScout 4.2 software 
Table 1. Types of pharmacophoric features and pharmacophore fit values of 17 training set compounds obtained by the LigandScout 4.2 software. Red, yellow, and purple indicate HBA, hydrophobic, and AR interaction, respectively

\begin{tabular}{|c|c|c|c|c|}
\hline No & Active compound name & Type & Matching features $\left.{ }^{\star}\right)$ & Pharmacophore fit \\
\hline 1 & 4-Hydroxytamoxifen & Training & & 44.59 \\
\hline 2 & Arzoxifene & Training & & 55.22 \\
\hline 3 & AZD9496 & Training & & 33.87 \\
\hline 4 & BHPI & Training & & 43.95 \\
\hline 5 & Brilanestrant & Training & & 43.60 \\
\hline 6 & C3D999 & Training & & 53.99 \\
\hline 7 & $\mathrm{E} 4 \mathrm{D} 600$ & Training & & 54.13 \\
\hline 8 & Elacestrant & Training & & 54.11 \\
\hline 9 & Ferutinin & Training & & 45.44 \\
\hline 10 & GW_5638 & Training & & 43.42 \\
\hline 11 & GW_7604 & Training & & 44.23 \\
\hline 12 & ICI_164384 & Training & & 44.83 \\
\hline 13 & Nafoxidine & Training & & 46.52 \\
\hline 14 & Raloxifene & Training & & 55.10 \\
\hline 15 & Raloxifene_D4 & Training & & 55.14 \\
\hline
\end{tabular}

Table 2. Screening results of 186 AIACS, its intermediate, and derivative compounds with pharmacophore model 2

\begin{tabular}{lc|c|c|c|c|c}
\hline No & Compound code & \multicolumn{3}{c}{ Pharmacophore features } & Pharmacophore fit \\
\hline 1 & $3 \mathrm{~B} 8$ & & & & & 53.43 \\
2 & $3 \mathrm{~B} 10$ & & & & & 53.39 \\
3 & $3 \mathrm{~B} 7$ & & & & & 53.39 \\
4 & $3 \mathrm{~A} 4$ & & & & & \\
5 & $3 \mathrm{~B} 5$ & & & & & 46.27 \\
6 & $3 \mathrm{~B} 2$ & & & & 46.22 \\
7 & $3 \mathrm{~B} 4$ & & & & & 46.20 \\
8 & $3 \mathrm{~B} 3$ & & & & 45.96 \\
9 & $3 \mathrm{~B} 1$ & & & & 45.95 \\
10 & $3 \mathrm{~A} 11$ & & & & & \\
11 & $3 \mathrm{~B} 9$ & & & & & 45.92 \\
12 & $3 \mathrm{~A} 12$ & & & & & \\
13 & $3 \mathrm{~B} 11$ & & & & & \\
14 & $3 \mathrm{~A} 6$ & & & & & \\
\hline
\end{tabular}

${ }^{{ }^{*}}$ Red, yellow, and purple indicated HBA, hydrophobic, and AR interaction, respectively

3B10, and 3B7 had the best pharmacophore fit values as indicated by their chemical features that are in harmony with the features of the tamoxifen pharmacophore model. None of the hits were Mannich base derivatives. The result was different from the result of in vitro evaluation against MCF-7 cell lines of the Mannich base of AMACs reported previously (active but nonselective) [8-9].

\section{Molecular Docking}

The structure of ERa in the complex with E4D600 ligands (PDB code: 1SJ0) was selected for in silico study because the parameters were suitable for experimental studies, with a resolution of $1.9 \AA$, free R-values of 0.272 , and working R-values of 0.218 . The R-value illustrates a measure of how well the enhanced structure predicts the 


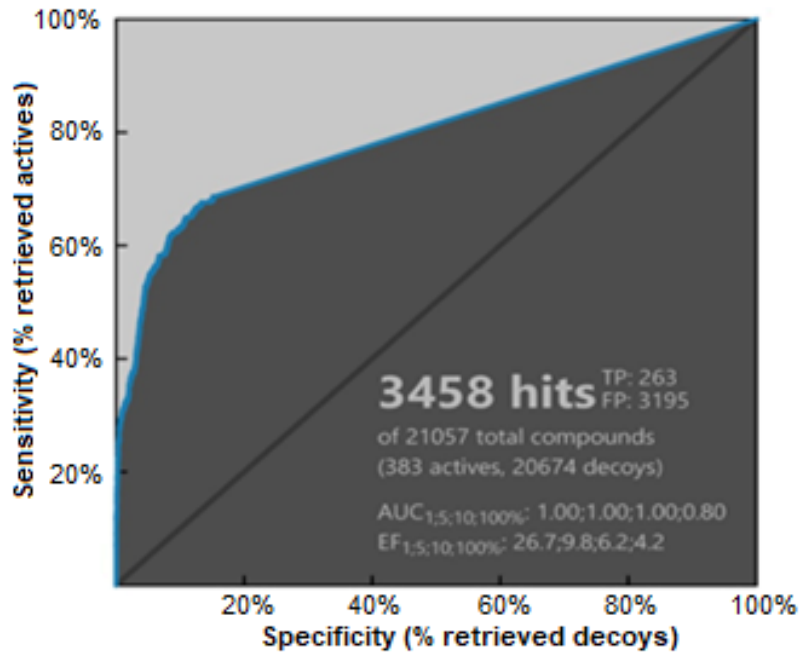

Fig 3. ROC curve model 2

observed data [30]. Interactions between co-crystalline ligand E4D600 with the active site of ERa were dominated by hydrophobic interactions with ARs, and hydrogen bonds with phenoxy and hydroxyl oxygen (Fig. 4 and 5). The best ligand-docking conformation is shown in Fig. 6 . The RMSD value of the copy ligand-docking conformation in the active site of ERa compared to the co-crystalline ligand-docking conformation at the same receptor was $0.940 \AA(<2.0 \AA)$, indicating the validity of the protocol.

The results of molecular docking of 14 hit compounds obtained from the ligand-based pharmacophore model are shown in Table 3. The free energy values, $\Delta \mathrm{G}$, of the 10 best hits (3A6, 3B1, 3B2, 3B3, 3B4 3B7, 3B8, 3B9, 3B10, and 3B11) did not differ significantly with that of tamoxifen. The interaction of amino acid residues with compounds 3B8 and 3B9 was 14 and 15. It was comparable with the interaction of amino acid with tamoxifen having 17 residues (Table S3 and Table 4). The hydrophobic interaction patterns of compounds 3B7, 3B8, 3B9, 3B10, and 3B11 showed similar triangular patterns and two little differences in the distance (Table S4 and Table 5).

\section{Drug Scan and Synthesis Accessibility Analysis}

The drug scan and synthesis accessibility analysis of 10 hit compounds were performed using molecular docking study by running them in www.swissadme.ch. The results showed that seven compounds (3A6, 3B1, $3 B 2,3 B 3,3 B 4,3 B 7$, and 3B11) fulfilled Lipinski's Rule of
Five (Table 6) and three compounds (3B8, 3B9, and 3B10) had $\log \mathrm{P}$ values higher than other ligands ( $\log \mathrm{P}$ $>5$ ), while the synthesis accessibility (SA) values ranged from 4.24 to 4.67 . The molecular weights of the ligands were in the range of 334.41-444.95 $\mathrm{g} / \mathrm{mol}$ which is higher than tamoxifen but still met Lipinski's Rule of Five (MW $<500 \mathrm{~g} / \mathrm{mol}$ ). The rule was a set of in silico guidelines applied to drug discovery to prioritize compounds with a

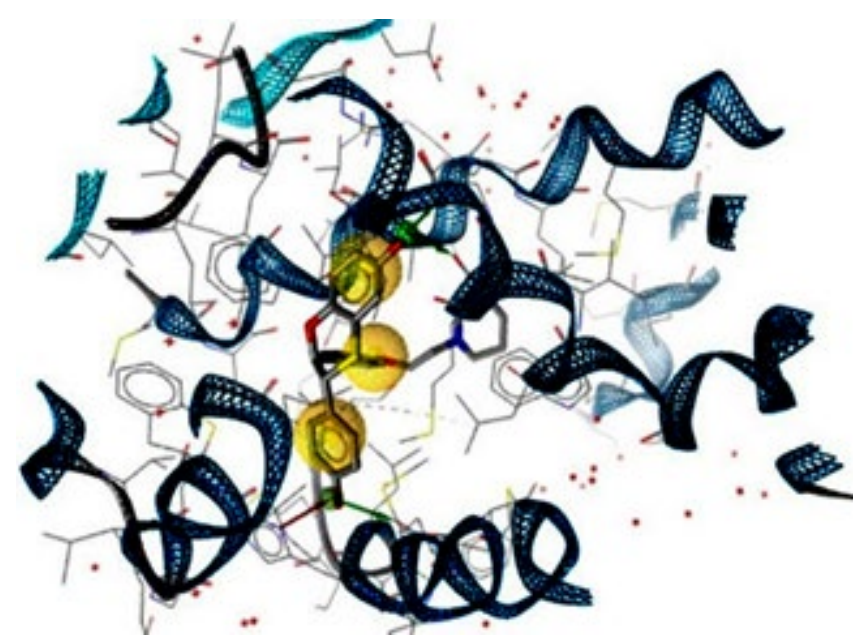

Fig 4. Pharmacophoric features between the native ligand $\mathrm{E} 4 \mathrm{D} 600$ with $\mathrm{ER}$ a derived from $\mathrm{X}$-ray derivative structures (PDB code: 1SJ0)

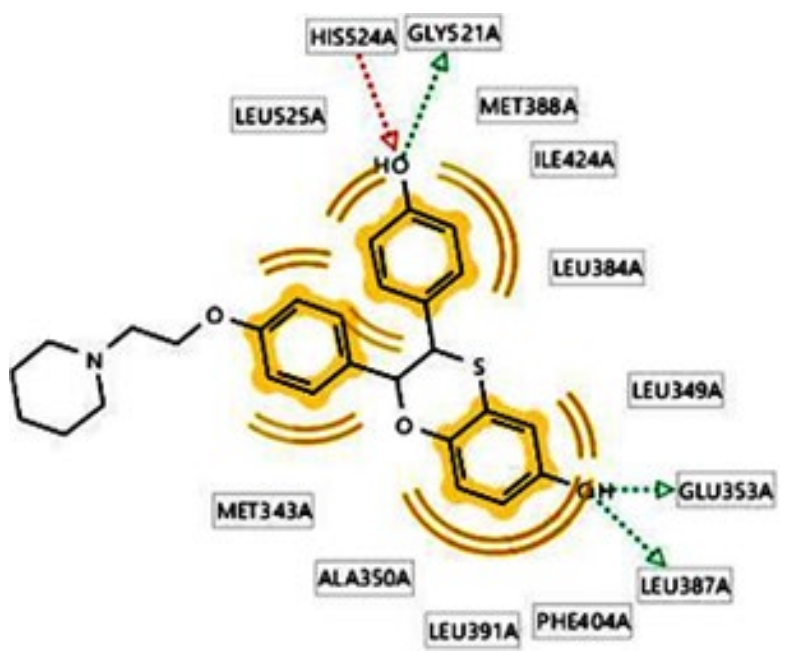

Fig 5. $2 \mathrm{D}$ structure visualization describes a hydrophobic bag in the form of a hydrophobic interaction of the native ligand $\mathrm{E} 4 \mathrm{D} 600$ with a residue at the receptor. Hydrophobic interactions, donor and acceptor hydrogen bonds are described as yellow balls, green, and red arrows, respectively 


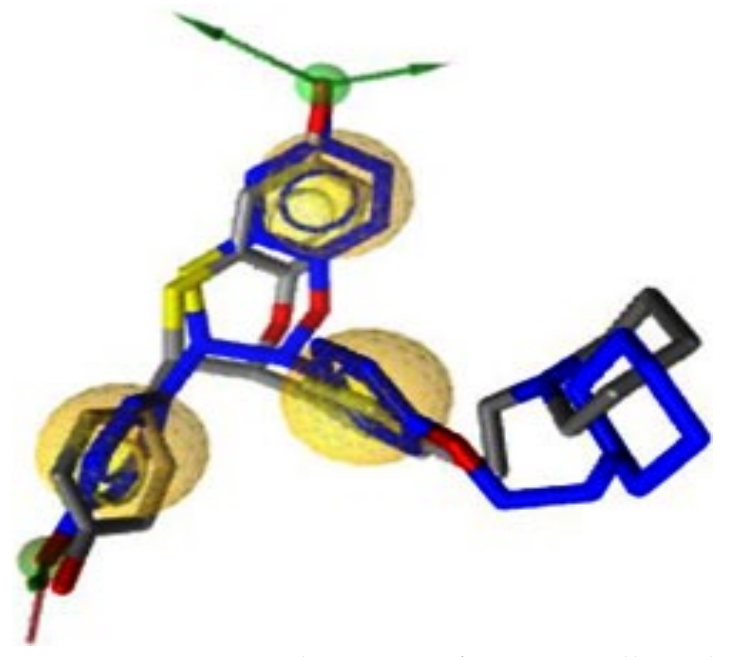

Fig 6. Superpose visualization of co-crystalline ligand (blue) with copy ligand using Autodock 4.2 integrated with Ligandscout 4.2
Table 3. Docking results of design compound molecules with estrogen receptors $\alpha$ (PDB code: $1 \mathrm{SJ} 0$ )

\begin{tabular}{ccc}
\hline No & Compound code & $\Delta \mathrm{G}(\mathrm{kcal} / \mathrm{mol})$ \\
\hline 1 & $3 \mathrm{~A} 4$ & -18.04 \\
2 & $3 \mathrm{~A} 6$ & -18.11 \\
3 & $3 \mathrm{~A} 11$ & -14.26 \\
4 & $3 \mathrm{~A} 12$ & -16.34 \\
5 & $3 \mathrm{~B} 1$ & -18.36 \\
6 & $3 \mathrm{~B} 2$ & -18.57 \\
7 & $3 \mathrm{~B} 3$ & -18.92 \\
8 & $3 \mathrm{~B} 4$ & -18.37 \\
9 & $3 \mathrm{~B} 5$ & -16.87 \\
10 & $3 \mathrm{~B} 7$ & -20.97 \\
11 & $3 \mathrm{~B} 8$ & -20.88 \\
12 & $3 \mathrm{~B} 9$ & -20.90 \\
13 & $3 \mathrm{~B} 10$ & -20.57 \\
14 & $3 \mathrm{~B} 11$ & -20.47 \\
15 & Tamoxifen & -19.87 \\
\hline
\end{tabular}

Table 4. Contact residues of 10 selected compounds and Tamoxifen

\begin{tabular}{|c|c|c|c|c|c|c|c|c|c|c|c|}
\hline \multirow{2}{*}{$\begin{array}{l}\text { Contact } \\
\text { residue }\end{array}$} & \multicolumn{11}{|c|}{ Compounds code } \\
\hline & $3 \mathrm{~A} 6$ & $3 \mathrm{~B} 1$ & $3 \mathrm{~B} 2$ & $3 \mathrm{~B} 3$ & 3B4 & $3 \mathrm{~B} 7$ & $3 \mathrm{~B} 8$ & $3 \mathrm{~B} 9$ & $3 \mathrm{~B} 10$ & $3 \mathrm{~B} 11$ & $\mathrm{Tam}^{*}$ \\
\hline Leu 525A & $\sqrt{ }$ & $\sqrt{ }$ & $\sqrt{ }$ & $\sqrt{ }$ & $\sqrt{ }$ & $\sqrt{ }$ & $\sqrt{ }$ & $\sqrt{ }$ & $\sqrt{ }$ & $\sqrt{ }$ & $\sqrt{ }$ \\
\hline Thr 347A & $\sqrt{ }$ & $\sqrt{ }$ & $\sqrt{ }$ & $\sqrt{ }$ & $\sqrt{ }$ & & & & & $\sqrt{ }$ & $\sqrt{ }$ \\
\hline $\operatorname{Trp} 383 \mathrm{~A}$ & $\sqrt{ }$ & $\sqrt{ }$ & $\sqrt{ }$ & $\sqrt{ }$ & $\sqrt{ }$ & $\sqrt{ }$ & $\sqrt{ }$ & $\sqrt{ }$ & $\sqrt{ }$ & $\sqrt{ }$ & $\sqrt{ }$ \\
\hline Leu 536A & & & $\sqrt{ }$ & & & $\sqrt{ }$ & $\sqrt{ }$ & $\sqrt{ }$ & $\sqrt{ }$ & & $\sqrt{ }$ \\
\hline Leu 354A & & & $\sqrt{ }$ & & & $\sqrt{ }$ & $\sqrt{ }$ & $\sqrt{ }$ & $\sqrt{ }$ & & $\sqrt{ }$ \\
\hline Ala $350 \mathrm{~A}$ & $\sqrt{ }$ & $\sqrt{ }$ & $\sqrt{ }$ & $\sqrt{ }$ & $\sqrt{ }$ & $\sqrt{ }$ & $\sqrt{ }$ & $\sqrt{ }$ & $\sqrt{ }$ & $\sqrt{ }$ & $\sqrt{ }$ \\
\hline Met 388A & $\sqrt{ }$ & $\sqrt{ }$ & $\sqrt{ }$ & & & $\sqrt{ }$ & $\sqrt{ }$ & $\sqrt{ }$ & $\sqrt{ }$ & $\sqrt{ }$ & $\sqrt{ }$ \\
\hline Leu 391A & $\sqrt{ }$ & & & $\sqrt{ }$ & $\sqrt{ }$ & $\sqrt{ }$ & $\sqrt{ }$ & $\sqrt{ }$ & $\sqrt{ }$ & $\sqrt{ }$ & $\sqrt{ }$ \\
\hline Phe $404 \mathrm{~A}$ & & & & $\sqrt{ }$ & $\sqrt{ }$ & & $\sqrt{ }$ & $\sqrt{ }$ & $\sqrt{ }$ & $\sqrt{ }$ & $\sqrt{ }$ \\
\hline Leu $428 \mathrm{~A}$ & $\sqrt{ }$ & & & & & $\sqrt{ }$ & $\sqrt{ }$ & $\sqrt{ }$ & $\sqrt{ }$ & $\sqrt{ }$ & $\sqrt{ }$ \\
\hline Leu $384 \mathrm{~A}$ & $\sqrt{ }$ & & & & & $\sqrt{ }$ & $\sqrt{ }$ & $\sqrt{ }$ & $\sqrt{ }$ & $\sqrt{ }$ & $\sqrt{ }$ \\
\hline Ile $424 \mathrm{~A}$ & $\sqrt{ }$ & $\sqrt{ }$ & $\sqrt{ }$ & & & & $\sqrt{ }$ & $\sqrt{ }$ & $\sqrt{ }$ & $\sqrt{ }$ & $\sqrt{ }$ \\
\hline Met $343 \mathrm{~A}$ & $\sqrt{ }$ & & $\sqrt{ }$ & & $\sqrt{ }$ & & $\sqrt{ }$ & $\sqrt{ }$ & & & $\sqrt{ }$ \\
\hline Phe $425 \mathrm{~A}$ & & & & & & & $\sqrt{ }$ & $\sqrt{ }$ & & & $\sqrt{ }$ \\
\hline His $524 \mathrm{~A}$ & $\sqrt{ }$ & $\sqrt{ }$ & $\sqrt{ }$ & & & & & & & & $\sqrt{ }$ \\
\hline Met $421 \mathrm{~A}$ & $\sqrt{ }$ & $\sqrt{ }$ & $\sqrt{ }$ & & & & & $\sqrt{ }$ & & $\sqrt{ }$ & $\sqrt{ }$ \\
\hline Leu $346 \mathrm{~A}$ & & $\sqrt{ }$ & & $\sqrt{ }$ & $\sqrt{ }$ & & $\sqrt{ }$ & $\sqrt{ }$ & & $\sqrt{ }$ & $\sqrt{ }$ \\
\hline Glu 353A & & & & $\sqrt{ }$ & & & & & & & \\
\hline Leu 349A & & & & $\sqrt{ }$ & $\sqrt{ }$ & & & & & $\sqrt{ }$ & \\
\hline Leu 387A & & & & $\sqrt{ }$ & $\sqrt{ }$ & $\sqrt{ }$ & & & $\sqrt{ }$ & $\sqrt{ }$ & \\
\hline Met $522 \mathrm{~A}$ & & & & & & $\sqrt{ }$ & $\sqrt{ }$ & $\sqrt{ }$ & $\sqrt{ }$ & & \\
\hline Leu $402 \mathrm{~A}$ & & & & & & & $\sqrt{ }$ & & & & \\
\hline
\end{tabular}

^Tam $=$ Tamoxifen

high probability of absorption [31]. In general, Lipinski's rules describe the solubility of certain compounds that affect the penetration of these compounds across cell membranes through passive diffusion [32]. This rule can 
Table 5. The distance between the pharmacophore features of the selected compounds. Distance A, B, and C refer to Fig. 1

\begin{tabular}{|c|c|c|c|c|}
\hline \multirow{2}{*}{ No } & \multirow{2}{*}{ Compounds code } & \multicolumn{3}{|c|}{ Distance $(\AA)$} \\
\hline & & $\mathrm{A}$ & B & $\mathrm{C}$ \\
\hline 1 & $3 \mathrm{~B} 7$ & 6.87 & 6.26 & 5.24 \\
\hline 2 & $3 \mathrm{~B} 8$ & 6.96 & 6.21 & 5.26 \\
\hline 3 & $3 \mathrm{~B} 9$ & 6.98 & 6.86 & 5.11 \\
\hline 4 & $3 \mathrm{~B} 10$ & 6.91 & 6.22 & 5.15 \\
\hline 5 & $3 \mathrm{~B} 11$ & 6.87 & 6.28 & 5.24 \\
\hline \multicolumn{2}{|c|}{ Average Distance $(\AA) \pm \mathrm{SD}$} & $6.92 \pm 0.051$ & $6.37 \pm 0.28$ & $5.20 \pm 0.066$ \\
\hline 6 & $\begin{array}{l}\text { Pharmacophores } \\
\text { Features of Model } 2\end{array}$ & 6.65 & 5.86 & 5.32 \\
\hline 7 & Tamoxifen & 6.37 & 5.21 & 4.91 \\
\hline $\begin{array}{l}\text { Differenc } \\
\text { compoun }\end{array}$ & $\begin{array}{l}\text { average distance of } \\
\text { Model } 2\end{array}$ & 0.27 & 0.81 & 0.12 \\
\hline $\begin{array}{l}\text { Differenc } \\
\text { compoun }\end{array}$ & $\begin{array}{l}\text { average distance of } \\
\text { Tamoxifen }\end{array}$ & 0.55 & 1.16 & 0.29 \\
\hline
\end{tabular}

Table 6. The prediction results based on Lipinski's rule of five and synthesis accessibility

\begin{tabular}{lcccccc}
\hline \multirow{2}{*}{$\begin{array}{c}\text { Compounds } \\
\text { code }\end{array}$} & $\begin{array}{c}\text { PW } \\
(\mathrm{g} / \mathrm{mol})\end{array}$ & $\begin{array}{c}\text { Log P } \\
\text { (Consensus) }\end{array}$ & $\begin{array}{c}\text { Hydrogen } \\
\text { Bond Acceptor }\end{array}$ & $\begin{array}{c}\text { Hydrogen } \\
\text { bond Donor }\end{array}$ & $\begin{array}{c}\text { TPSA } \\
(\AA)\end{array}$ & $\begin{array}{c}\text { Synthesis } \\
\text { Accessibility }\end{array}$ \\
\cline { 2 - 6 } 3A6 & 394.46 & 3.56 & 5 & 2 & 72.31 & 4.60 \\
3B1 & 334.41 & 3.59 & 3 & 2 & 53.85 & 4.26 \\
3B2 & 348.44 & 3.88 & 3 & 2 & 53.85 & 4.37 \\
3B3 & 368.86 & 4.13 & 3 & 2 & 53.85 & 4.24 \\
3B4 & 352.40 & 3.85 & 4 & 2 & 53.85 & 4.23 \\
3B7 & 410.15 & 4.64 & 3 & 1 & 45.06 & 4.52 \\
3B8 & 424.53 & 5.20 & 3 & 1 & 45.06 & 4.64 \\
3B9 & 444.95 & 5.41 & 3 & 1 & 45.06 & 4.51 \\
3B10 & 428.50 & 5.19 & 4 & 1 & 45.06 & 4.53 \\
3B11 & 440.53 & 4.95 & 4 & 0 & 54.29 & 4.67 \\
Tamoxifen & 371.51 & 5.77 & 2 & 12.47 & 3.01 \\
\hline
\end{tabular}

also be used to predict the pharmacokinetics of a compound as a drug candidate [33].

The SA values of the 10 hits ranged from 4.23 to 4.67, which indicated that the synthesis difficulty was medium and there were no differences among the compounds. However, compounds 3B1, 3B2, 3B3, and 3B4 with SA values in the range of 4.23-4.37 were easier to synthesize than the others. The SA values were based on the analysis of structural fragments of more than 13 million compounds, assuming that the more numerous the molecular fragments, the more difficult the molecules are to prepare. Descriptors correct this fragmental contribution method for molecular size and complexity and the SA values range from 1 (easily synthesized) to 10 (difficult to be synthesized) [34].

\section{- CONCLUSION}

One hundred and eighty-six AIACs, AMACs, intermediates, and their Mannich base derivative compounds were successfully screened using ligandbased pharmacophore modeling, and the hits obtained were further screened using structure-based molecular docking in the active site of ER $\alpha$, and were analyzed using drug scan and synthesis accessibility. Seven 
compounds namely $3 \mathrm{~A} 6,3 \mathrm{~B} 1,3 \mathrm{~B} 2,3 \mathrm{~B} 3,3 \mathrm{~B} 4,3 \mathrm{~B} 7$, and $3 \mathrm{~B} 11$ were suggested to be potentially active as ERa inhibitors and deserve to be synthesized and further investigated.

\section{- SUPPORTING INFORMATION}

Table S1.a-g: Structures of the 186 Asymmetrical Hexahydro-2H-Indazole Analogs of Curcumin (AIACs), AMACs, its intermediate, and Mannich base derivative compounds; Table S2.a: Four native ligands of ERa receptor; Table S2.b.: Data of 30 ERa inhibitor compounds Table S3: 2D and 3D visualization results of 10 selected compounds and Tamoxifen docked at 1 SJ0 receptors; Table S4: 2D and 3D visualization of chemical features with triangular patterns.

\section{- ACKNOWLEDGMENTS}

This work was supported by the Doctoral Program Dissertation Grant Universitas Indonesia, No.: NKB0092/UN2.R3.1/HKP.05.00/2019, which was gratefully appreciated.

\section{- AUTHOR CONTRIBUTIONS}

HYT conducted the experiment; HY, AY and KMD supervised the experiment; HYT, HY, and AY wrote and revised the manuscript. All authors agreed to the final version of this manuscript.

\section{- REFERENCES}

[1] Mellatyar, H., Talaei, S., Nejati-Koshki, K., and Akbarzadeh, A., 2016, Targeting HSP90 gene expression with 17-DMAG nanoparticles in breast cancer cells, Asian Pac. J. Cancer Prev., 17 (5), 24532457.

[2] Bray, F., Ferlay, J., Soerjomataram, I., Siegel, R.L., Torre, L.A., and Jemal, A., 2018, Global cancer statistics 2018: GLOBOCAN estimates of incidence and mortality worldwide for 36 cancers in 185 countries, CA Cancer J. Clin., 68 (6), 394-424.

[3] Caldon, C.E., 2014, Estrogen signaling and the DNA damage response in hormone dependent breast cancer, Front. Oncol., 4, 1-9.

[4] Hayashi, S.I., Eguchi, H., Tanimoto, K., Yoshida, T., Omoto, Y., Inoue, A., Yoshida, N., and Yamaguchi,
Y., 2003, The expression and function of estrogen receptor alpha and beta in human breast cancer and its clinical application, Endocr. Relat. Cancer, 10 (2), 193-202.

[5] den Hollander, P., Savage, M.I., and Brown, P.H., 2013, Targeted therapy for breast cancer prevention. Front. Oncol., 3, 250.

[6] Revalde, J.L., Li, Y., Hawkins, B.C., Rosengren, R.J., and Paxton, J.W., 2015, Heterocyclic cyclohexanone monocarbonyl analogs of curcumin can inhibit the activity of ATP-binding cassette transporters in cancer multidrug resistance, Biochem. Pharmacol., 93 (3), 305-317.

[7] Yerdelen, K.O., Gul, H.I., Sakagami, H., and Umemura, N., 2015, Synthesis and biological evaluation of 1, 5-bis (4-hydroxy-3-methoxyphenyl) Penta-1, 4-dien-3-one and its aminomethyl derivatives, J. Enzyme Inhib. Med. Chem., 30 (3), 383-388.

[8] Kurnia, A., Saputri, F.C., and Hayun, H., 2019, Synthesis and anticancer potential of aminomethyl derivatives of methyl-substituted asymmetrical curcumin mono-carbonyl, J. Appl. Pharm. Sci., 9 (8), 18-24.

[9] Prasetyaningrum, P.W., Bahtiar, A., and Hayun, H., 2018, Synthesis and cytotoxicity evaluation of novel asymmetrical mono-carbonyl analogs of curcumin (AMACs) against Vero, HeLa, and MCF7 cell lines, Sci. Pharm., 86, 25.

[10] Bala, S., Sharma, N., Kajal, A., Kamboj, S., and Saini, V., 2014, Mannich bases: An important pharmacophore in present scenario, Int. J. Med. Chem., 2014, 191072.

[11] Bayomi, S.M., El-Kashef, H.A., El-Ashmawy, M.B., Nasr, M.N.A., El-Sherbeny, M.A., Badria, A., Abouzeid, L.A., Ghaly, M.A., Abdel-Aziz, N.I., 2013, Synthesis and biological evaluation of new curcumin derivatives as antioxidant and antitumor agents, Med. Chem. Res., 22 (3), 1147-1162.

[12] Bayomi, S.M., El-Kashef, H.A., El-Ashmawy, M.B., Nasr, M.N.A., El-Sherbeny, M.A., Abdel-Aziz, N.I., El-Sayed, M.A.A., Suddek, G.M., El-Messery, S.M., and Ghaly, M.A., 2015, Synthesis and biological 
evaluation of new curcumin analogues as antioxidant and antitumor agents: Molecular modeling study, Eur. J. Med. Chem., 101, 584-594.

[13] Reddy, M.T., Rddy, V.H., Reddy R.C.K., Reddy, V.K., and Reddy, Y.V.R., 2010, Synthesis and molecular docking studies of new substituted indazole derivatives for anti-breast cancer activity, Der Pharma Chem., 6 (6), 411-417.

[14] Plescia, S., Raffa, D., Plescia, F., Casula, G., Maggio, B., Daidone, G., Raimondi, M.V., Cusimano, M.G., Bombieri, G., and Meneghetti, F., 2010, Synthesis and biological evaluation of new indazole derivatives, ARKIVOC, 10, 163-177

[15] Shin, W.H., Zhu, X., Bures, M.G., and Kihara, D., 2015, Three-dimensional compound comparison methods and their application in drug discovery, Molecules, 20 (7), 12841-12862.

[16] Li, Q., Chen, J., Luo, S., Xu, J., Huang, Q., and Liu, T., 2015, Synthesis and assessment of the antioxidant and antitumor properties of asymmetric curcumin analogues, Eur. J. Med. Chem., 93, 461-469.

[17] Wolber, G., and Langer, T., 2005, LigandScout: 3-D pharmacophores derived from protein-bound ligands and their use as virtual screening filters, $J$. Chem. Inf. Model., 45 (1), 160-169.

[18] Seidel, T., Ibis, G., Bendix, F., and Wolber, G., 2010, Strategies for 3D pharmacophore-based virtual screening, Drug Discovery Today Technol., 7 (4), e221-e228.

[19] Kar, S., and Roy, K., 2013, How far can virtual screening take us in drug discovery?, Expert Opin. Drug Discovery, 8 (3), 245-261.

[20] Maruthanila, V.L., Elancheran, R., Roy, N.K., Bhattacharya, A., Kunnumakkara, A.B., Kabilan, S., and Kotoky, J., 2019, In silico molecular modelling of selected natural ligands and their binding features with estrogen receptor alpha, Curr. Comput. Aided Drug Des., 15 (1), 89-96.

[21] Afreen, S., Uddin, N., Mehjabin, K.Z., Niketa, T.K., Nesa, F., Akther, S.,Akther, S., Chakraborty, S., Chowdhury D., and Akther, N., 2018, In silico molecular docking approach of some selected isolated phytochemicals from Phyllanthus emblic against breast cancer, BJSTR, 10 (2), 7661-7665.

[22] Banegas Luna, A.J., Cerón-Carrasco, J.P., and Pérez-Sánchez, H., 2018, A review of ligand-based virtual screening web tools and screening algorithms in large molecular databases in the age of big data, Future Med. Chem., 10 (22), 2641-2648

[23] Sun, Y., Zhou, H., Zhu, H., and Leung, S.W., 2016, Ligand-based virtual screening and inductive learning for identification of SIRT1 inhibitors in natural products, Sci. Rep., 6 (1), 19312.

[24] Liu, C., Yin, J., Yao, J., Xu, Z., Tao, Y., and Zhang, H., 2020, Pharmacophore-based virtual screening toward the discovery of novel anti-echinococcal compounds, Front. Cell. Infect. Microbiol., 10, 118.

[25] Qing, X., Lee, X.Y., De Raeymaecker, J., Tame, J., Zhang, K., De Maeyer, M., and Voet, A., 2014, Pharmacophore modeling: Advances, limitations, and current utility in drug discovery, J. Recept. Ligand Channel Res., 7, 81-92.

[26] Lone, M.Y., Manhas, A., Athar, M., and Jha, P.C., 2018, Identification of InhA inhibitors: A combination of virtual screening, molecular dynamics simulations, and quantum chemical studies, J. Biomol. Struct. Dyn., 36 (11), 2951-2965.

[27] Yuniarti, N., Mungkasi, S., Yuliani, S.H., and Istyastono, E.P., 2019, Development of a graphical user interface application to identify marginal and potent ligands for estrogen receptor alpha, Indones. J. Chem., 19 (2), 531-537.

[28] Setiawati, A., Riswanto, F.D.O., Yuliani, S.H., and Istyastono, E.P.2014, Retrospective validation of a structure-based virtual screening protocol to identify ligands for estrogen receptor alpha and its application to identify the alpha-mangostin binding pose, Indones. J. Chem., 14 (2), 103-108.

[29] Mysinger, M.M., Carchia, M., Irwin, J.J., and Shoichet, B.K., 2012, Directory of useful decoys, enhanced (DUD-E): Better ligands and decoys for better benchmarking, J. Med. Chem., 55 (14), 65826594.

[30] Kim, S., Wu, J.Y., Birzin, E.T., Frisch, K., Chan, W., Pai, L.Y., Yang, Y.T., Mosley, R.T., Fitzgerald, P.M.D., Sharma, N., Dahllund, J., Thorsell, A.G., 
DiNinno, F., Rohrer, S.P., Schaeffer, J.M., and Hammond, M.L., 2004, Estrogen receptor ligands. II. Discovery of benzoxathiins as potent, selective estrogen receptor alpha modulators, J. Med. Chem., 47 (9), 2171-2175.

[31] Doak, B.C., Over, B., Giordanetto, F., and Kihlberg, J., 2014, Oral druggable space beyond the rule of 5: Insights from drugs and clinical candidates, Chem. Biol., 21 (9), 1115-1142.

[32] Lipinski, C.A., Lombardo, F., Dominy, B.W., and Feeney, P.J., 1997, Experimental and computational approaches to estimate solubility and permeability in drug discovery and development settings, $A d v$. Drug Delivery Rev., 23 (1-3), 3-25.

[33] Benet, L.Z., Hosey, C.M., Ursu, O., and Oprea, T.I., 2016, BDDCS, the rule of 5 and drugability, $A d v$. Drug Delivery Rev., 101, 89-98.

[34] Daina, A., Michielin, O., and Zoete, V., 2017, SwissADME: A free web tool to evaluate pharmacokinetics, drug-likeness and medicinal chemistry friendliness of small molecules, Sci. Rep., 7,42717 . 